\title{
D oença de Behçet cursando com trombose de veia cava superior
}

\author{
Behçet's disease presenting with superior vena cava thrombosis \\ O tacílio Figueiredo da Silva Júnior ${ }^{1}$, Ricardo H enrique de Sousa Araújo², \\ Eutília Andrade M edeiros Freire', Ronaldo Rangel Travassos Júnior ${ }^{4}$, \\ Thiago Emanuel Rodrigues Cavalcante ${ }^{2}$, Tarcísio José Pinheiro Lucena ${ }^{2}$, \\ Norberto de Castro N ogueira Neto ${ }^{5}$, Alessandra Vanessa de Albuquerque $\mathrm{M} \mathrm{elo}^{5}$
}

\begin{abstract}
Resumo
A trombose venosa é o principal acometimento do sistema vascuIar na doença de Behçet, sendo uma complicação freqüente na evolução clínica dessa patologia, e está mais associada ao sexo masculino. O s autores relatam o caso de uma paciente do sexo feminino que apresentou início da doença há 3 anos com úlceras orais, além de úlceras genitais, e que vem cursando com episódios recorrentes de trombose venosa, sendo a primeira uma trombose venosa profunda no membro inferior direito; a segunda, de jugular interna esquerda; e, por último, uma trombose da veia cava superior.
\end{abstract}

Palavras-chave: D oença de Behçet, trombose venosa, síndrome da veia cava superior.

A doença de Behçet é uma doença inflamatória multissistêmica de evolução crônica, cujos sinais e sintomas podem aparecer demodo insidioso ou, ainda, de forma abrupta, com acometimento de múltiplos órgãos ${ }^{1,2}$. O s episódios se caracterizam por remissões e exacerbações inesperadas, ten den do a uma diminuição da freqüência e dos episódios com o tempo ${ }^{3}$.

\footnotetext{
1. Professor adjunto, Disciplina Segunda Clínica Cirúrgica, Universidade Federal da Paraíba (UFPB), João Pessoa, PB.

2. Acadêmico de Medicina, UFPB, João Pessoa, PB.

3. Professora assistente, Departamento de Medicina Interna, UFPB. João Pessoa, PB.

4. Professor adjunto, Disciplina de Pneumologia, UFPB, João Pessoa, PB. 5. Médico(a) radiologista.

Artigo submetido em 03.02.06, aceito em 20.03.06.
}

\begin{abstract}
Venous thrombosis is the main hazard to the vascular system in Behçet's disease. It is a frequent complication throughout the clinical evolution of this illness, which is moreassociated with the malegender. The authors report the case of a female patient with disease onset 3 years ago, showing oral and genital ulcers presenting with recurring episodes of venous thrombosis. The first one was a deep venous thrombosis in the right leg; the second was in the left internal jugular vein; and finally a superior vena cava thrombosis.
\end{abstract}

Key words: Behçet's disease, venous thrombosis, superior vena cava syndrome.

$\mathrm{N}$ ão há testes laboratoriais ou histopatológios que possam fornecer o diagnóstico de certeza da doença de Behçet, e, portanto, esteérealizado através de avaliação clínica ${ }^{4}$, que, apesar denão evidenciar sintoma característico, baseia-se na avaliação de critérios de uso comum. Para este diagnóstico, observa-se ocorrência de úlcerasorais recorrentes, associadas a duas das seguintes manifestações: uveítes, úlceras genitais, lesões cutâneas, lesões vasculares, artrite e meningoencefalite. Além disso, podemos contar ainda com a reação de patergia (hiper-reatividade cutânea a traumas mecânicos repetitivos), ressaltando que é um teste que apresenta uma baixa sensibilidade, além de ser inespecífico ${ }^{1,5}$.

A pesar da etiologia não estar totalmente esclarecida, inúmeros estudos trazem evidências de que 0 H LA-B51 está bastante associado à patologia. Ainda ocorrem na literatura relatos sobre a rel ação de antí- 
genos estreptocócicos, a ativação das células T e a patogênese da doença ${ }^{6}$.

\section{Relato de caso}

Paciente do sexo feminino, 22 anos, solteira, internada em hospital após apresentar queixa de edema em região cervical, facial eem tórax superior e vasos dilatados na região torácica anterior e superior (Figura 1), de início súbito há 3 dias. Realizou tomografia computadorizada (TC) detórax, a qual evidenciou aumento do calibre da veia cava superior, com imagem hipoatenuanteno seu interior sugerindo trombo, além de alargamento mediastinal, imagens deconsolidação parenquimatosa nos segmentos basais dos lobos pulmonares inferiores, broncogramas aéreos de permeio e derrame pleural bilateral (Figura 2). A TC cervical evidencia aumento das partes moles do pescoço, sugerido pelo espessamento do plano cutâneo eborramento do plano subcutâneo, além de circulação colateral pela veiajugular externa direita, musculatura torácica e paravertebral (Figura 3), estendendo-se inferiormente para a região da veia ázigos. D iante dos exames, fica claro o diagnóstico de síndrome da veia cava superior.

A pacienteteveos primeiros sintomas da doença de Behçet há 3 anos, com 0 aparecimento de estomatites ulceradas de repetição e de úlcera genital, tendo sido feito exame histopatológico dessa lesão, que revelou apenas processo inflamatório crônico e inespecífico, tendo recebido o diagnóstico clínico. Seguiu fazendo uso de talidomida 500 mg, havendo, ainda, após o

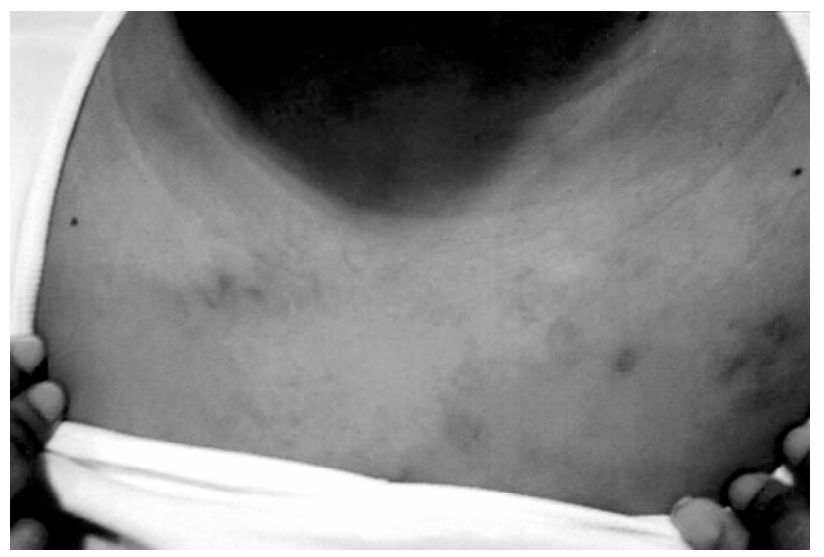

Figura 1 - Paciente apresentando circulação colateral visível em região torácica anterior

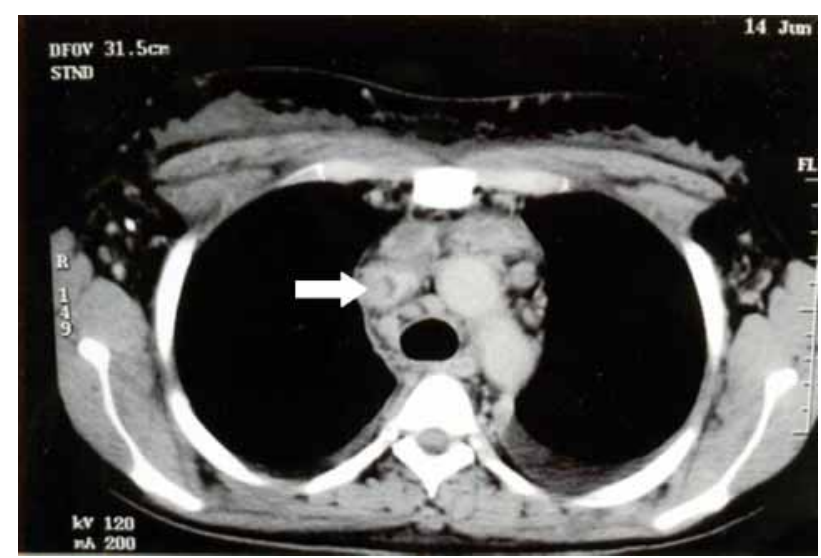

Figura 2 - Tomografia computadorizada (TC) mostrando imagem hipoatenuante no interior da veia cava superior, correspondendo a trombo (seta); presença de linfonodos mediastinais e derrame pleural bilateral

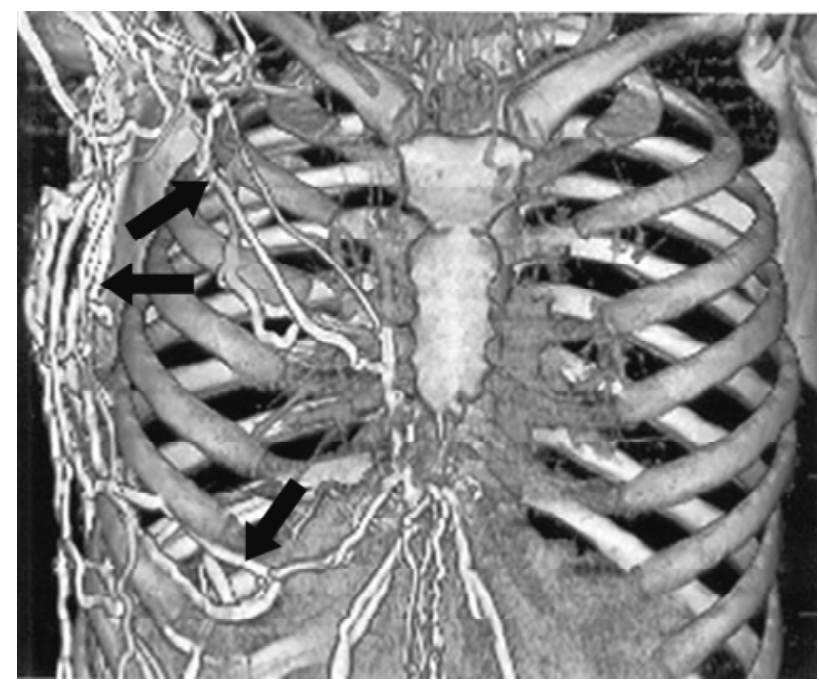

Figura 3 - Reconstrução com técnica volume rendering demonstrando circulações colaterais na parede torácica (setas)

início do tratamento, recorrência de úlcera genital por várias vezes. $\mathrm{H}$ á 2 anos, teve diagnóstico por duplex scan de trombose venosa profunda no membro inferior direito e, há 3 meses, teve um episódio de edema cervical, tendo sido diagnosticado novamente através do duplex scan como uma trombose da veia jugular interna esquerda, quando começou o tratamento ininterrupto com anticoagulanteoral sob monitorização da relação internacional normalizada ou international normalized ratio(IN R ), o quenão impediu o aparecimento do quadro atual de trombose da veia cava superior. 
A pósinternamento hospitalar, a anticoagulação oral foi substituída pela heparina de baixo peso molecular por via subcutânea, devido à possibilidade cogitada de realização de pulsoterapia. Foram solicitados testes laboratoriais para confirmação da atividade da doença (proteína C reativa, velocidade dehemossedimentação, eletroforese de proteínas séricas), com resultados normais. Face aos resultados negativos dos testes laboratoriaise evolução favorável (regressão do edema eda dor), optou-se pela não-realização da pulsoterapia durante esse episódio agudo.

A paciente segue fazendo tratamento de manutenção com warfarina e vem evoluindo com remissão progressiva do edema de face e pescoço. N o momento, a paciente apresenta recidiva de úlceras orais e genitais, com proposta de realização de pulsoterapia com ciclofosfamida, devido à reativação da doença.

\section{D iscussão}

A decisão de descrição do caso citado deveu-se, ao nosso ver, à particularidade da situação, visto que a paciente é originária de área onde a doença não é freqüente (a doença de B ehçet é rara nas Américas e Europa ${ }^{5,7}$ ), cursando com quadro vascular raro (a freqüência de acometimento da veia cava superior é de apenas $6 \%$ dos casos de trombose nesta doença $\left.{ }^{8}\right)$, ainda mais sendo do sexo feminino (a trombose venosa profunda é uma complicação de maior ocorrência no sexo masculino ${ }^{3,9-11}$ ), além de já estar em anticoagulação no momento do acometimento pelo presente quadro vascular.

$H$ á dados, na literatura, de que a trombose de veia cava é um acometimento que confere a esta doença um prognóstico sombrio ${ }^{12}$, apesar de outras publicações científicas descreverem que ospacientes, vítimas dessa complicação, podem ter uma sobrevida longa, desde que desenvolvam uma circulação colateral superficiall1.

A anticoagulação aparececomo propostaterapêutica de eventos trombóticos em pacientes com doença de Behçet em diversos trabalhos $5^{5,13-16}$, apesar de constar na literatura a possibilidade de recorrências de trombose com essa terapêutica, acreditando-se que seja devido à inflamação vascular edisfunção endotelial subjacente. A duração dessa terapia ainda é discutida7,12. Embora a pacientejá se encontrasse em tratamento com anticoagulante oral desde a ultima oclusão venosa, houve necessidade de substituição pela administração de he- parina debaixo peso molecular por via subcutânea, pela possibilidade preten dida de real ização de pulsoterapia.

A talidomida éuma proposta terapêutica freqüente na literatura para o tratamento e a prevenção das recidivas das úlceras orais e genitais, tendo sido, inclusive, submetida a estudo randomizado, duplo-cego, com controle por placebo, que evidenciou a eficácia semelhante da droga administrada nas dosagens de $100 \mathrm{mg}$ e $300 \mathrm{mg}^{17}$. Entretanto, o fármaco não é um agente modificador da doença, pois há recorrência das úlceras com a descontinuidade da terapêutica ${ }^{6,17-19}$.

O s corticosteróides sistêmicos têm o uso indicado nos quadros deuveíte, al ém de acometimentos vasculares e neurológicos agudos; entretanto, não são indicados para o tratamento de manutenção a longo prazo, pelo risco de efeitos colaterais $s^{7,12}$. A pesar desses dados, um único trabalho relata que essas drogas ainda podem ser usadas, por períodos prolongados, associadas aos anticoagulantes, objetivando a preven ção dequadros de trombose venosa ${ }^{20}$.

0 uso da ciclofosfamida está indicado para o acometimento ocular, conferindo remissões prolongadas, al ém de acometimentos vasculares e neurológicos, podendo ser realizado através de tratamento oral diário ou de pulsos endovenosos mensalmente $e^{7,11,20}$.

0 tratamento na doença de Behçet varia de acordo com as manifestações clínicas do paciente e com suas gravidades, requerendo quase sempre uma abordagem multidisciplinar ${ }^{7}$. Apesar do amplo arsenal disponível para o tratamento dessa patologia, nenhuma das opções parece ser ideal, visto que não são capazes de controlar 0 aparecimento dos sintomas ${ }^{7,18}$.

\section{Referências}

1. International Study G roup of Behcet's D isease. Evaluation of diagnostic (classification) criteria in Behcet's disease: towards internationally agreed criteria. Br J Rheumatol. 1992;31: 229-308.

2. Radke PW, Schwarz ER, G roesdonk H , G raf J, Janssens U . Thrombosis in Behcet's disease: report of a casefollowed by a systematic review using the methodology of the evidence based medicine. J Thromb Thrombolysis. 2001;11:137-41.

3. Al-O taibi LM, Porter SR, Poate TW. Behcet's disease: a review. J D ent Res. 2005;84:209-22.

4. Dervis E, Geyik N. Sensitivity and specificity of different diagnostic criteria for Behcet's disease in a group of T urkish patients. J D ermatol. 2005;32:266-72. 
5. Cecil RL, Goldman L, Bennet JC. Doenças músculoesqueléticas e do tecido conjuntivo: doença de Behçet. In: G oldman L, Bennet JC, editor. T ratado de medicina interna. Rio de Janeiro: G uanabara Koogan; 2001. p. 1716-7.

6. H irohata S, Kikuchi H. Behcet's disease. Arthritis R es Ther. 2003;5:139-46.

7. Leiba M, Ehrenfeld M. Behcet's disease: current therapeutic perspectives. Curr Treat O ptions Cardiovasc M ed. 2005;7:139-48.

8. C astelli P, C aronno R, Pifaretti G, et al. Endovascular treatment for superior vena cava obstruction in Behcet disease. J V asc Surg. 2005;41:548-51.

9. H ouman M H, Ben G horbel I, Khiari Ben Salah I, Lamloum $M$, Ben Ahmed M, M iled M. Deep vein thrombosis in Behcet's disease. Clin Exp Rheumatol. 2001;19(5 Suppl 24):S48-50.

10. Ames PR, Steuer A, Pap A, D enman AM. Thrombosis in Behcet's disease: a retrospective survey from a single UK centre. Reumathology (O xford). 2001;40:652-5.

11. Carreño Pérez L. Enfermedad de Behçet. An M ed Interna (M adrid). 2001;18:401-4.

12. KontogiannisV, Powell RJ. Behcet's disease. Postgrad M ed J. 2000;76:629-37.

13. ChaloupkaK, Baglivo $E, H$ ofer $M$, et al. Thrombosedes sinus veineux cerébraux dans la maladie: description d'un cas et revue de la litérature. Klin $\mathrm{M}$ onatsbl Augenheilkd. 2003;220:186-8.

14. W echsler B, Sbai A, D u-Boutin LT, D uhaut P, D ormont D, Piette JC. M anifestations neurologiques de la maladie de Behçet. Rev N eurol (Paris). 2002;158:926-33.
15. Cemri M, Erkan A, O zdemir M, Cengel A. Behcet's disease with alargeand freeright atrial thrombus. Eur J Echocardiogr. 2002;3:233-5.

16. H ouman H, Lamloum M, Ben G horbel I, Khiari-Ben Salah I, M iled M. Thromboses caves dans la maladie de Behçet. Analyse d'unesérie de 10 observations. Ann Med Interne (Paris). 1999;150:587-90.

17. Ehrlich, GE. B ehcet diseaseand theemergence of thalidomide. Ann Intern M ed. 1998;128;494-5.

18. H amuryudan V, M at C, Saip $S$, et al. Thalidomide in the treatment of the mucocutaneous lesions of the Behcet syndrome: a randomized, double-blind, placebo-controlled trial. Ann Intern M ed. 1998;128:443-50.

19. D emiroglu H, Yalcin S, Buyukasik Y. M ucocutaneouslesions in the Behcet syndrome. Ann Intern M ed. 1998;129:836.

20. Vidaller Palacín A, Robert O lalla J, Sanuy Jiménez $B$, et al. T ratamiento de la enfermedad de Behçet. An M ed Interna (M adrid). 2002;19:594-8.

Correspondência:

O tacílio Figueiredo da Silva Júnior

Av. D om Pedro II, 1172, Centro

CEP 58013-420 - João Pessoa, PB

Tel.: (83) 3222.4912/4048 / (83) 9927.9244

Fax: (83) 3221.1336

E-mail: otaciliofigueiredo@terra.com.br 